\title{
¿Por qué los estudiantes de la Universidad Pedagógica ISFODOSU escogen la carrera de Educación?
}

\author{
Why ISFODOSU Pedagogical University students choose \\ the education career?
}

\author{
Emmanuel Silvestre ${ }^{a}$ ORCID: 0000-0002-9958-4848 \\ Vladimir Figueroa-Gutiérrez $z^{\mathbf{b}}$ ORCID: 0000-0003-0944-3572 \\ José Vicente Díaz-Esteve ${ }^{c}$ ORCID: 0000-0003-1586-5552
}

Recibido: 19/10/2019 • Aprobado: 23/1/2020

\begin{abstract}
Cómo citar: Silvestre, E., Figueroa-Gutiérrez, V., \& Vicente Díaz-Esteve, J. (2020). ¿¿Por qué los estudiantes de la Universidad Pedagógica ISFODOSU escogen la carrera de educación? Ciencia y Educación, 4(1), 47-69. Doi: https://doi.org/10.22206/cyed. 2020.v4i1.pp47-69
\end{abstract}

\section{Resumen}

Como parte de una investigación dirigida a establecer los perfiles de los estudiantes de nuevo ingreso en ISFODOSU, incluimos variables vocacionales para determinar las motivaciones más importantes para la elección de carrera de los participantes, así como para la elección de esta universidad y la opinión sobre sus recursos tecnológicos. La muestra $(N=744)$ fue elegida al azar, de modo proporcional entre los estratos Recintos, Carreras y Sexo. En primer lugar, validamos la escala FIT-Choice con dos análisis factoriales confirmatorios. Los 12 factores motivacionales mostraron un buen nivel de ajuste $(\mathrm{RMSEA}=.062$; CFI $=.92$ y TLI $=.901)$, al igual que los 6 factores perceptuales (RMSEA $=.062$; $\mathrm{CFI}=.944 \mathrm{y}$ TLI $=$.924). La confiabilidad $(\alpha)$ de los factores motivacionales fue buena, entre .66 y .93 , al igual que la de los factores perceptuales, que estuvo entre .73 y .92. Las motivaciones más importantes para elegir la carrera de Educación fueron influir en el futuro de niños, hacer una contribución social y el gusto por enseñar. Entre los factores perceptuales estuvieron la satisfacción con la elección, la exigencia de la carrera y la exigencia de la profesión.

\begin{abstract}
As part of research aimed at establishing the profiles of students of new entry at ISFODOSU, we include vocational variables to determine the most important motivations for the choice of career of the participants, as well as for the election of this university and opinion on its technological resources. The sample (No. 744) was chosen at random, proportionally among the Enclosures, Careers and Sex strata. First, we validate the FIT-Choice scale with two confirmatory factorial analyses. The 12 motivational factors showed a good level of fit (RMSEA .062; CFI .92 and TLI .901, as well as the 6 perceptual factors (RMSEA .062; CFI .944 and TLI .924). The reliability of the motivational factors was good, between .66 and .93 , as were the perceptual factors between .73 and .92. The most important motivations for choosing the education career were to influence the future of children, make a social contribution and a taste for teaching. Among the perceptual factors were satisfaction with the choice, the demand for the career and the demand of the profession. The most important factors in choosing ISFODOSU as
\end{abstract}

\footnotetext{
a Instituto Superior de Formación Docente Salomé Ureña, República Dominicana. Correo-e: esilvestre@esilvestre.com

b Instituto Superior de Formación Docente Salomé Ureńa, República Dominicana. Correo-e: vladimir.figueroa@isfodosu.edu.do

c Universitat de València, España. Correo-e: diazjv@uv.es
} 
Los factores más importantes para elegir ISFODOSU como universidad fueron la calidad, el factor económico y los servicios. Los recursos tecnológicos de la universidad fueron considerados como muy adecuados y actualizados. Usando análisis de varianza de tres factores aparecieron algunas diferencias entre los estratos. Se detallan los recintos, las carreras y los sexos que necesitaban mejorar sus motivaciones y actitudes frente a la universidad.

Palabras clave: elección de carrera; escala FIT-Choice; motivación del profesorado; validación escala.

\section{Introducción}

Este reporte forma parte de una investigación más amplia que busca establecer los perfiles socioeconómicos, culturales y psicosociales de los estudiantes de nuevo ingreso en el Instituto Superior de Formación Docente Salomé Ureña (ISFODOSU). Conocer los perfiles de los futuros docentes en términos de rendimiento académico, nivel socioeconómico, capital cultural y estructura familiar es muy importante para la planificación de modelos eficaces de formación docente, puesto que muchos investigadores han señalado que los antecedentes familiares y sociales de los estudiantes tienen una estrecha relación con el rendimiento académico (Cervini et al., 2014; Creemers \& Kyriakides, 2007; Creemers et al., 2010; Hanushek, 2016; Kyriakides et al., 2019; Patrinos, 2007; Reynolds et al., 2011).

En esta investigación se incluyeron datos demográficos, hábitos de estudio, uso y preferencias de medios de comunicación y redes sociales, hábitos de recreación, uso y conocimientos sobre recursos tecnológicos, así como el aspecto socioafectivo de los estudiantes. En este aspecto, se midieron expectativas como la autoestima, la autoeficacia y el locus de control, pero los resultados que reportamos aquí se refieren específicamente a las motivaciones expresadas por los ingresados recientemente en la universidad para seleccionar Educación como su carrera profesional y para seleccionar ISFODOSU como universidad.

Estas motivaciones incluyeron sus expectativas sobre la formación universitaria y los factores que fueron importantes para elegir ISFODOSU como universidad, así como su autopercepción sobre su vocación a university were quality, economics and services. The university's technological resources were considered to be very adequate and up-to-date. Using three-factor variance analysis, some differences between the strata appeared. It details the venues, careers and sexes that needed to improve their motivations and attitudes towards the university.

Keywords: Career choice; FIT-Choice scale; teacher motivation; validation scale.

y competencia profesional, y sus expectativas y temores sobre su futuro profesional. Para medir estos datos vocacionales administramos, además de algunas preguntas ad hoc específicas, la escala de factores que influyen en la elección de los estudios de Educación (Factors Influencing Teaching-Choice), FIT-Choice, por sus siglas en inglés, siguiendo el método de su validación en España por Gratacós \& López-Jurado (2016).

\section{Factores que influyen en la elección de los estudios de Educación}

Los resultados de las investigaciones acerca de los factores que influyen en la elección de la carrera docente por parte de los estudiantes universitarios son diversos. Entre los principales factores se identifican: a) utilidad social de la enseñanza (Lin et al., 2012), b) interés por trabajar con niños y jóvenes (Bruinsma \& Jansen, 2010; Frei et al., 2017), y c) programas de formación docente con altos estándares de calidad que se reflejen en los resultados académicos de los estudiantes (Bergmark et al., 2018; Han \& Yin, 2016). Efectivamente, los países con mejores puntuaciones en los informes PISA de 2009 y 2012 fueron aquellos en donde la profesión docente tiene mayor prestigio (Gratacós \& López-Jurado, 2016). Además de este prestigio social, también son mencionados como motivadores las influencias familiares y las experiencias previas de enseñanza (Calvo, 2006).

Por el contrario, entre los factores que influyen negativamente en el prestigio de la carrera docente se encuentran: a) la baja calidad de la formación, la falta de articulación entre teoría y práctica (Guerriero 
\& Deligiannidi, 2017; Vaillant \& Marcelo, 2012), b) la insatisfacción de la sociedad hacia la profesión docente (Ávalos et al., 2010; Elacqua et al., 2018; Martínez-de-la-Hidalga \& Villardón-Gallego, 2018). El bajo prestigio social hacia la carrera docente influye negativamente en la atracción de los estudiantes más talentosos (Calvo, 2006; García-Poyato \& Cordero, 2019; Prats, 2016).

Sin embargo, hasta que Watt \& Richardson (2007) desarrollaron el modelo de base para la escala FIT-Choice no había un consenso teórico que explicara la relevancia e importancia relativa de los diferentes factores que llevan a un estudiante a escoger la docencia como profesión.

Desde un principio, este modelo ha sido adaptado, traducido y aplicado en diferentes contextos nacionales en Australia (Watt \& Richardson, 2007), Holanda (Fokkens-Bruinsma \& Canrinus, 2012), EE. UU., Alemania y Noruega (Watt et al., 2012), Turquía (Eren \& Tezel, 2010; Topkaya \& Uztosun, 2012), Canadá y Omán (Klassen et al., 2011), Suiza (Berger \& D’Ascoli, 2012), Croacia (Jugoviæ et al., 2012) y China (Lin et al., 2012). Ya mencionamos que Gratacós \& López-Jurado (2016) tradujeron y replicaron las características psicométricas de la escala FIT-Choice en España. En la región Caribe de Colombia lo hicieron Said-Hung et al. (2017). En República Dominicana la escala fue validada por Tomás (2014).

El modelo FIT-Choice, de Watt \& Richardson (2007), surge de la Teoría de la expectativa del valor (Wigfield \& Eccles, 2000), la cual explica la ejecución de logros y elecciones a través del valor que se espera obtener de las propias acciones. Así, los determinantes para elegir una carrera serían las expectativas de éxito y la evaluación de la tarea.

El modelo FIT-Choice está compuesto por tres tipos de motivaciones: extrínsecas, intrínsecas y altruistas. Las motivaciones extrínsecas serían las influencias de amigos y familiares, las condiciones laborales y la posibilidad de proseguir otro tipo de estudios, entre otras. Las motivaciones intrínsecas serían el agrado por trabajar con niños, el deseo de ayudar a la gente, la capacidad de influenciar, el interés por enseñar, las relaciones interpersonales, el prestigio social y otras similares. Las motivaciones altruistas serían aquellas relativas a hacer contribuciones sociales, mejorar la equidad en la sociedad y el interés por influir en los niños con vistas a su futuro.

La Figura 1 permite visualizar las relaciones entre los diferentes factores del modelo FIT-Choice. En la parte superior de la figura se colocan elementos socializadores previos a la elección de carrera, como la disuasión social, la percepción de experiencias previas de enseñanza y las influencias sociales (de amigos, familiares y profesores).

Figura 1. Modelo teórico del FIT-Choice empíricamente validado

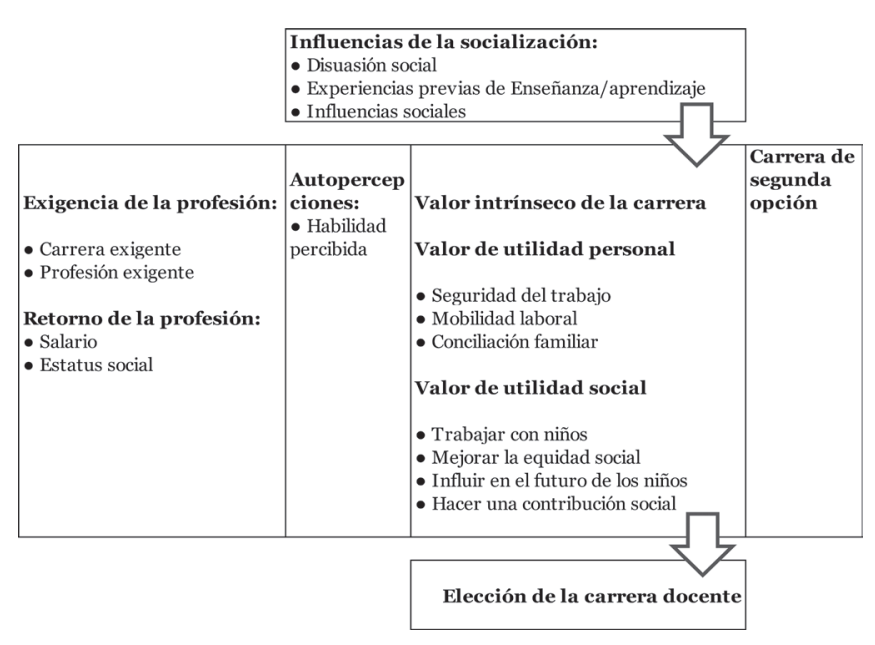

Fuente: Modelo de FIT-Choice extraído de Gratacós \& LópezJurado (2016, p. 8).

Los determinantes principales de la motivación se encuentran en la parte central de la figura y se clasifican en cuatro: expectativas y creencias sobre la profesión (exigencias de la profesión y retorno de la profesión), autopercepción (habilidad percibida para enseñar), valor intrínseco de la tarea o carrera (utilidad personal o utilidad social) y carrera de segunda opción (si la educación fue elegida en primer lugar o no).

En la Figura 1 podemos advertir que las expectativas y creencias de la profesión se dividen en dos: por una parte, se encuentra la exigencia que implica el estudio de la carrera y lo exigente de ejercitarla como profesión, y por la otra, el retorno que implica ejercer la profesión, en términos de estatus social y salario. 
El valor de la tarea tiene tres vertientes. En primer lugar, se encuentra el valor intrínseco de la carrera, que equivale al gusto por enseñar. En segundo lugar, tenemos el valor de utilidad personal, constituido por la seguridad del trabajo, la movilidad laboral y la conciliación familiar. En tercer lugar, está el valor de utilidad social: mejorar la equidad social, trabajar con niños y/o adolescentes, hacer una contribución social e influir en el futuro de los niños. Por último, se considera la propia satisfacción con la elección de la carrera docente.

\subsection{Objetivos}

Los objetivos específicos de este reporte fueron:

2.1.1. Determinar la validez y la confiabilidad de la escala FIT-Choice en nuevos estudiantes de Pedagogía en nuestro país para garantizar la adecuación y la precisión de los resultados sobre los demás objetivos.

2.1.2. Establecer la importancia relativa de los factores motivacionales y perceptuales de la escala FIT-Choice para seleccionar Educación como carrera.

2.1.3. Determinar los factores que motivaron al estudiante de nuevo ingreso a la selección del ISFODOSU como universidad.

2.1.4. Determinar la opinión de los participantes sobre la cantidad y la adecuación de los recursos tecnológicos de ISFODOSU.

2.1.5. Establecer la influencia de las variables independientes Recinto, Plan de Estudios y Sexo en las motivaciones, percepciones y opiniones de los participantes.

\section{Método}

Para este trabajo se seleccionó una muestra de ingresados, incluyendo los que ingresaron condicionalmente en la condición de nivelación. Esta condición se otorga temporalmente a aquellos solicitantes que necesitan reforzar algunos contenidos de la prueba de admisión académica. Esta muestra fue seleccionada entre los aprobados en las pruebas de 2018, cuya población fue de 1,688 en el momento de la selección.

Se seleccionó al azar una muestra representativa y estratificada de esa población. Para un nivel de confianza de $95 \%$ y un margen de error de $\pm 2.69 \%$, nuestra muestra representativa contó con 744 participantes. La escala FIT-Choice se administró dentro del cuestionario general de la investigación.

Para la estratificación de la muestra de la encuesta fueron usadas tres variables independientes:
3.1. Recinto
Eugenio María de Hostos (EMH) 15\%
Emilio Prud'Homme (EPH) 10\%
Félix Evaristo Mejía (FEM) 18\%
Juan Vicente Moscoso (JVM) 13\%
Luís Napoleón Núñez Molina (LNNM) 25\%
Urania Montás (UM) 19\%

\subsection{Sexo}

Femenino 60\% (Educación Física 19\%)

Masculino 40\% (Educación Física 81\%)

3.3. Plan de estudios o carrera

Educación Inicial 13\%

Educación Primaria 23\%

Educación Secundaria 32\%

Educación Física 32\%

De acuerdo con las proporciones de estudiantes para esas variables independientes, los casos se muestran en la Tabla 1. Varias casillas quedaron vacías en esta tabla porque había recintos que no tenían todas las carreras, una carrera solo tenía un sexo, o la proporción de un sexo no fue significativa.

Tabla 1. Muestra de ingresados

\begin{tabular}{lcccccccc}
\hline & \multicolumn{1}{c}{ Educ. } & & & & & & \\
Muestra & Física & Secundaria & Primaria & Inicial & \\
\hline Recintos & F & M & F & M & F & M & F & TOTAL \\
EMH & 29 & 83 & & & & & & 112 \\
EPH & & & 38 & 17 & 8 & & 13 & 76 \\
FEM & & & 78 & 26 & 11 & & 18 & 133 \\
JVM & & & & & 46 & 26 & 27 & 99 \\
LNNM & 15 & 61 & 46 & 31 & 7 & & 23 & 183 \\
UM & 12 & 38 & & & 55 & 19 & 17 & 141 \\
\hline & & & & & & & & \\
TOTAL & 56 & 182 & 162 & 74 & 127 & 45 & 98 & 744 \\
\hline
\end{tabular}

Fuente: elaboración propia. 
Las variables dependientes que conciernen a este reporte fueron:

A. Demográficas: Sexo, Recinto, Plan de estudios (tres ítems).

1. Motivaciones: factores para seleccionar la carrera de Educación, 38 ítems (FIT-Choice). Los ítems de la escala Fit-Choice se contestaron en una escala de uno a siete, donde uno significaba Nada importante y siete significaba Muy importante.

2. Autopercepción sobre vocación y competencia. Temores y expectativas respecto del futuro profesional, 20 ítems (FIT-Choice).

3. Factores para seleccionar ISFODOSU, nueve ítems. Los ítems con los factores para seleccionar ISFODOSU fueron siete. Los tres factores más importantes para el participante debían ser ordenados por él en orden de importancia. Hubo además dos ítems sobre la actitud frente a los recursos tecnológicos de la universidad a ser contestados en escalas tipo Likert, donde uno representaba el total desacuerdo y cinco el total acuerdo.

\subsection{Procedimiento}

La muestra de ingresados fue seleccionada con la ayuda de los Servicios estudiantiles de cada uno de los recintos. El Departamento de Investigación les suministró a los Servicios estudiantiles las cuotas de la muestra para cada sexo y carrera en cada recinto. Los Servicios estudiantiles reclutaron entonces estas cuotas de participantes y los condujeron a los laboratorios de Informática de cada recinto para llenar nuestra encuesta. Esta encuesta había sido cargada en la plataforma SurveyMonkey.

\section{Resultados}

\subsubsection{Validez de construcción de la escala de los factores que influyen en la elección de los estudios de Educación}

El cuestionario FIT-Choice incluye 12 factores motivacionales entre 38 ítems, cuatro factores sobre creencias perceptuales sobre la educación, con 14 ítems, y dos factores perceptuales sobre la decisión tomada, con seis ítems. Por razones prácticas, siguiendo la metodología utilizada por Gratacós \& López-Jurado (2016) y Tomás (2014), realizamos dos Análisis Factoriales Confirmatorios (AFC), uno para los 12 factores motivacionales y otro para los seis factores perceptuales.

En la Tabla 2 mostramos varios índices en común entre nuestro estudio y los autores que acabamos de mencionar, para comparar la bondad de ajuste de los datos con el modelo motivacional de 12 factores. El RMSEA, según sus siglas en inglés, es la raíz del error de aproximación al cuadrado y nos indica si el modelo basado en la muestra representa a la población. Como vemos en la tabla, el RMSEA de nuestro estudio presente cumplió el criterio de encontrarse entre .05 y .08, más ampliamente que en los otros dos estudios con que lo comparamos.

Tabla 2. Índice de la bondad de ajuste de los datos con el modelo de 12 factores motivaciones según estudio y criterio

\begin{tabular}{lccc}
\hline Estudio & RMSEA & CFI & TLI \\
Criterio & $.05=>.08$ & $>.9$ & $90 \%$ \\
Gratacós \& López-Jurado, 2016 & .052 & .952 & .959 \\
Tomás, 2014 & .04 & .98 & N/D \\
ISFODOSU, 2018 (presente) & .062 & .92 & .901 \\
\hline
\end{tabular}

Fuente: elaborado a partir de Gratacós \& López-Jurado (2016), Tomás (2014) y datos del presente estudio.

El índice de ajuste comparativo (CFI, por sus siglas en inglés) analiza el ajuste del modelo examinando la discrepancia entre los datos y el modelo hipotético. Este índice se considera adecuado si supera el criterio de .9. En la Tabla 2 podemos ver que el CFI que encontramos superó este criterio y fue completamente comparable al encontrado en los estudios de comparación.

El índice Tucker Lewis (TLI, por sus siglas en inglés) que encontramos indica que el modelo mejora el ajuste en un $90 \%$ con relación al modelo de la hipótesis nula, cumpliendo el criterio exigido por este índice. El estudio de Gratacós \& López-Jurado 
(2016) encontró un TLI un poco más alto y el estudio de Tomás (2014) no reportó este índice.

Nuestro Chi-cuadrado para el modelo de 12 factores motivacionales evalúa el ajuste general y la discrepancia entre las matrices de covarianza de la muestra y la ajustada, y resultó muy significativo, $\chi^{2}(599)=2208.349, p=.000$. El modelo se compuso de 88 variables, 38 de ellas observadas.

En la Tabla 3 presentamos las mismas comparaciones con estos índices, pero esta vez con relación al modelo de seis factores perceptuales. El REMSEA cumplió adecuadamente el criterio por encima del índice encontrado por Gratacós \& López-Jurado (2016) y comparable al encontrado por Tomás (2014).

Tabla 3. Índice de la bondad de ajuste de los datos con el modelo de 6 factores perceptuales según estudio y criterio

\begin{tabular}{lccc}
\hline Estudio & RMSEA & CFI & TLI \\
Criterio & $.05=>.08$ & $>.9$ & $90 \%$ \\
Gratacós \& López-Jurado, 2016 & .051 & .96 & 0.951 \\
Tomás, 2014 & .06 & .97 & N/D \\
ISFODOSU, 2018 (presente) & .062 & .944 & .924 \\
\hline
\end{tabular}

Fuente: Elaborado a partir de Gratacós \& López-Jurado (2016), Tomás (2014) y datos del presente estudio.
Como se aprecia en la tabla, el CFI encontrado en nuestro estudio es comparable con el de Tomás (2014) y más alto que el de Gratacós \& López-Jurado (2016). El TLI que encontramos para los factores perceptuales es comparable con el de Gratacós \& López-Jurado (2016), superando ambos el criterio de $90 \%$. Tomás (2014) no reportó este índice.

El Chi-cuadrado que encontramos para el modelo de seis factores perceptuales fue también muy significativo, $\chi^{2}(155)=590.843, p=.000$. El modelo se compuso de 46 variables, 20 de ellas observadas.

Ambos modelos, el de 12 factores motivacionales y el de seis factores perceptuales, mostraron un buen ajuste con los datos, por lo cual realizamos el análisis de confiabilidad de cada factor usando el índice Alfa de Cronbach. Todos los estimados de saturación factorial de los ítems con su factor propuesto fueron mayores de .40, siendo el más bajo .552. La gran mayoría pasaba de .70 . La confiabilidad para prácticamente todos los factores fue excelente.

\subsubsection{Confiabilidad de la escala FIT-Choice}

En la Tabla 4 se pueden encontrar las medias, las desviaciones estándares (DE) y las cargas factoriales de cada ítem motivacional, así como el Alfa de Cronbach para cada factor.

Tabla 4. Medias, desviaciones estándares y cargas factoriales de cada ítem motivacional, así como el Alfa de Cronbach para cada factor

\begin{tabular}{|c|c|c|c|c|c|}
\hline No. & Factores & Media & $\mathrm{DE}$ & Cargas factoriales & Alfa \\
\hline 1 & Habilidad percibida (HP) & 5.8 & 1.34 & & .9 \\
\hline B5. & Tengo cualidades para ser buen maestro/a. & 6 & 1.41 & .862 & \\
\hline B19. & Tengo buenas habilidades para enseñar. & 5.8 & 1.419 & .883 & \\
\hline B43. & La enseñanza es una profesión que se adecúa a mis habilidades. & 5.8 & 1.559 & .861 & \\
\hline 2 & Valor intrínseco de la carrera (VI) & 6 & 1.31 & & .81 \\
\hline B1. & Me interesa la enseñanza. & 6.3 & 1.408 & .784 & \\
\hline B7. & Siempre he querido ser maestro/a. & 5.3 & 1.783 & .664 & \\
\hline B12. & Me gusta enseńar. & 6.2 & 1.403 & .888 & \\
\hline 3 & Carrera de segunda opción (SO) & 2.3 & 1.515 & & .66 \\
\hline B11. & No tenía claro qué carrera quería estudiar. & 2.6 & 2.061 & .587 & \\
\hline B35. & No fui aceptado en la carrera de primera opción. & 2.3 & 1.984 & .6 & \\
\hline
\end{tabular}




\begin{tabular}{|c|c|c|c|c|c|}
\hline No. & Factores & Media & $\mathrm{DE}$ & Cargas factoriales & Alfa \\
\hline B48. & La carrera de Educación era la última opción que tenía. & 2 & 1.75 & .699 & \\
\hline 4 & Seguridad del trabajo (ST) & 5 & 1.674 & & .85 \\
\hline B14. & La enseñanza me ofrecerá un trabajo estable. & 5.3 & 1.811 & .813 & \\
\hline B27. & Ser maestro me permite tener un sueldo fijo. & 4.8 & 1.983 & .813 & \\
\hline B38. & La enseñanza es un trabajo seguro. & 5 & 1.898 & .813 & \\
\hline 5 & Conciliación familiar (CF) & 3.8 & 1.432 & & .81 \\
\hline B2. & $\begin{array}{l}\text { Por el horario escolar, trabajar como maestro me permitiría tener más tiempo para la } \\
\text { familia. }\end{array}$ & 4.6 & 1.868 & .587 & \\
\hline B4. & Siendo maestro tengo más vacaciones. & 2.9 & 1.94 & .552 & \\
\hline B16. & Los horarios me permitirán compatibilizarlo con mis responsabilidades familiares. & 4.7 & 1.869 & .748 & \\
\hline B18. & Siendo maestro tendré una jornada laboral corta. & 2.9 & 1.781 & .641 & \\
\hline B29. & Las vacaciones escolares encajan con mis obligaciones familiares. & 3.9 & 2.023 & .805 & \\
\hline 6 & Movilidad laboral (ML) & 4.5 & 1.626 & & .74 \\
\hline B8. & La enseñanza puede darme la oportunidad de trabajar en el extranjero. & 4.6 & 1.991 & .662 & \\
\hline B22. & La titulación de maestro tiene un reconocimiento en todas partes. & 4.9 & 1.92 & .749 & \\
\hline B45. & La enseńanza me permitirá elegir dónde quiero vivir. & 3.9 & 2.085 & .669 & \\
\hline 7 & Influir en el futuro de nińos/adolescentes (FNA) & 6.3 & 1.213 & & .87 \\
\hline B9. & La enseñanza me permitirá formar en valores a niños/adolescentes. & 6.5 & 1.251 & .869 & \\
\hline B23. & La enseñanza me permitirá influir en la próxima generación. & 6.3 & 1.318 & .836 & \\
\hline B53. & La enseńanza me permitirá influir en los niños/adolescentes. & 6.1 & 1.435 & .825 & \\
\hline 8 & Mejorar la equidad social (ME) & 5.6 & 1.442 & & .78 \\
\hline B36. & La enseńanza me permitirá elevar las ambiciones de la juventud desfavorecida. & 5.3 & 1.906 & .599 & \\
\hline B49. & La enseñanza me permitirá ayudar a las personas socialmente desfavorecidas. & 5.8 & 1.598 & .819 & \\
\hline B54. & La enseñanza me permitirá trabajar contra la desventaja social. & 5.7 & 1.639 & .834 & \\
\hline 9 & Hacer una contribución social (CS) & 6.3 & 1.21 & & .85 \\
\hline B6. & La enseńanza me permite dar un servicio a la sociedad. & 6.4 & 1.234 & .854 & \\
\hline B20. & Los maestros hacen una contribución valiosa a la sociedad. & 6.5 & 1.256 & .891 & \\
\hline B31. & Ser maestro me permite devolver a la sociedad lo que he recibido. & 5.9 & 1.587 & .741 & \\
\hline 10 & Trabajar con niños/adolescentes (TNA) & 5.5 & 1.644 & & .93 \\
\hline B13. & Quiero un trabajo que suponga trabajar con nińos/adolescentes. & 5.4 & 1.79 & .868 & \\
\hline B26. & Quiero trabajar en un entorno con nińos/adolescentes. & 5.6 & 1.763 & .93 & \\
\hline B37. & Me gusta trabajar con nińos/adolescentes. & 5.7 & 1.674 & .931 & \\
\hline 11 & Experiencias previas de enseńanza/aprendizaje (EP) & 5.6 & 1.321 & & .89 \\
\hline B17. & He tenido profesores a los que he admirado y me han influido positivamente. & 5.9 & 1.692 & .885 & \\
\hline B30. & He tenido profesores que han sido buenos modelos. & 6 & 1.661 & .891 & \\
\hline B39. & He tenido experiencias de aprendizaje positivas. & 6 & 1.468 & .817 & \\
\hline 12 & Influencias sociales (IS) & 4.1 & 1.908 & & .85 \\
\hline B3. & Mis amigos piensan que debería ser maestro/a. & 3.6 & 2.192 & .763 & \\
\hline B24. & Mi familia piensa que debería ser maestro/a. & 4.6 & 2.129 & .83 & \\
\hline B40. & La gente con la que he trabajado piensa que debería ser maestro/a. & 4.3 & 2.17 & .831 & \\
\hline
\end{tabular}

Fuente: elaboración propia. 
En la Tabla 5 se pueden encontrar las medias, las ítem perceptual, así como el Alfa de Cronbach para desviaciones estándares y las cargas factoriales de cada cada factor perceptual.

Tabla 5. Medias, desviaciones estándares y cargas factoriales de cada ítem perceptual, así como el Alfa de Cronbach para cada factor perceptual

\begin{tabular}{|c|c|c|c|c|c|}
\hline No. & Factores & Media & $\mathrm{DE}$ & Cargas factoriales & Alfa \\
\hline 1 & Carrera exigente (CE) & 6.1 & 1.24 & & .86 \\
\hline C10. & ¿Crees que la enseńanza exige un alto nivel de conocimiento? & 6.2 & 1.37 & .774 & \\
\hline C14. & ¿Crees que los maestros necesitan altos niveles de conocimiento técnico? & 6 & 1.38 & .882 & \\
\hline C15. & ¿Crees que los maestros necesitan un conocimiento altamente especializado? & 6 & 1.44 & .83 & \\
\hline 2 & Profesión exigente (PE) & 6 & 1.3 & & .76 \\
\hline C2. & ¿Crees que los maestros tienen una fuerte carga de trabajo? & 5.6 & 1.7 & .591 & \\
\hline C7. & ¿Crees que la enseñanza requiere un gran trabajo emocional? & 6.2 & 1.34 & .814 & \\
\hline C11. & ¿Crees que la enseñanza es un trabajo exigente? & 6.1 & 1.52 & .742 & \\
\hline 3 & Estatus social (ES) & 5.2 & 1.3 & & .86 \\
\hline C4. & ¿Crees que a los maestros se les percibe como profesionales? & 5.7 & 1.66 & .73 & \\
\hline C5. & ¿Crees que los maestros tienen un alto nivel de entusiasmo? & 5.2 & 1.53 & .614 & \\
\hline $\mathrm{C} 8$. & ¿Crees que la enseñanza se considera una profesión de alto estatus? & 5.3 & 1.73 & .708 & \\
\hline C9. & ¿Crees que los maestros se sienten valorados por la sociedad? & 4.9 & 1.74 & .756 & \\
\hline C12. & ¿Crees que la profesión de maestro está bien considerada? & 5 & 1.68 & .774 & \\
\hline C13. & ¿Crees que los maestros creen que su trabajo tiene un alto estatus social? & 5.1 & 1.62 & .709 & \\
\hline 4 & Salario (SA) & 4.4 & 1.6 & & .73 \\
\hline C1. & ¿Crees que los maestros están bien pagados? & 4.3 & 1.81 & .682 & \\
\hline C3. & ¿Crees que el ejercicio de la enseñanza está bien remunerado? & 4.5 & 1.66 & .849 & \\
\hline 5 & Disuasión social (DS) & 4.5 & 1.8 & & .75 \\
\hline D2. & ¿Te animaron a elegir otras carreras antes que la de Educación? & 4.6 & 2.18 & .664 & \\
\hline D4. & ¿Te dijeron otras personas que ser maestro no era una buena elección de carrera? & 4.4 & 2.17 & .664 & \\
\hline D6. & ¿Otras personas te influyeron para que consideraras otras alternativas a Educación? & 4.4 & 2.1 & .798 & \\
\hline 6 & Satisfacción con la elección (SE) & 6.2 & 1.3 & & .92 \\
\hline D1. & ¿En qué medida has considerado seriamente tu decisión de ser maestro? & 6.1 & 1.45 & .778 & \\
\hline D3. & ¿En qué medida estás satisfecho con tu decisión de ser maestro? & 6.1 & 1.41 & .969 & \\
\hline D5. & ¿En qué medida estás contento con tu decisión de ser maestro? & 6.2 & 1.39 & .93 & \\
\hline
\end{tabular}

Fuente: elaboración propia.

54 - Ciencia y Educación 2020; 4(1, enero-abril): 47-69 • Artículos de Investigación 


\subsubsection{Correlaciones entre los factores}

En la Tabla 6 ofrecemos las correlaciones estandarizadas entre los factores motivacionales. En esta tabla los nombres de los factores están representados por las abreviaturas que aparecen entre paréntesis en la Tabla 4.

Tabla 6. Correlaciones estandarizadas entre los factores motivacionales del FIT-Choice

\begin{tabular}{llllllllllll}
\hline & HP & VI & SO & ST & CF & ML & FNA & ME & CS & TNA & EP \\
HP & - & & & & & & & & & & \\
VI & .93 & - & & & & & & & & & \\
SO & -.23 & -.37 & - & & & & & & & & \\
ST & .49 & .41 & .29 & - & & & & & & & \\
CF & .40 & .29 & .51 & .79 & - & & & & & & \\
ML & .53 & .40 & .38 & .92 & .88 & - & & & & & \\
FNA & .87 & .93 & -.29 & .50 & .32 & .49 & - & & & & \\
ME & .77 & .76 & -.16 & .52 & .43 & .58 & .88 & - & & & \\
CS & .88 & .94 & -.26 & .50 & .34 & .49 & 1.03 & .85 & - & & \\
TNA & .75 & .73 & -.14 & .49 & .41 & .49 & .70 & .62 & .71 & - & \\
EP & .76 & .79 & -.19 & .42 & .34 & .46 & .76 & .67 & .81 & .63 & - \\
IS & .43 & .32 & .35 & .56 & .61 & .68 & .29 & .36 & .31 & .43 & .36 \\
\hline
\end{tabular}

Fuente: elaboración propia.

En la Tabla 7 ofrecemos las correlaciones estandarizadas entre los factores perceptuales. En esta tabla los nombres de los factores están representados por las abreviaturas que aparecen entre paréntesis en la Tabla 5.

Tabla 7. Correlaciones estandarizadas entre los factores perceptuales del FIT-Choice

\begin{tabular}{llllll}
\hline & CE & PE & ES & SA & DS \\
CE & - & & & & \\
PE & .89 & - & & & \\
ES & .61 & .59 & - & & \\
SA & .38 & .37 & .70 & - & \\
\hline
\end{tabular}

\begin{tabular}{lccccl}
\hline & CE & PE & ES & SA & DS \\
DS & .31 & .32 & .20 & .23 & - \\
SE & .74 & .70 & .54 & .36 & .26 \\
\hline
\end{tabular}

Nota: elaboración propia.

\subsection{Importancia de los factores motivacionales}

Según se puede observar en la Figura 2, las motivaciones más importantes para nuestros participantes escoger la carrera de Educación fueron influir en el futuro de niños y adolescentes, hacer una contribución social, el valor intrínseco de la carrera y la habilidad percibida. La motivación menos importante de todas fue elegir Educación como carrera de segunda opción, aunque también aparecen bajas las motivaciones Conciliación familiar, Influencias sociales y Movilidad laboral.

Figura 2. Importancia de los factores motivacionales para elegir la carrera de Educación para el total de la muestra

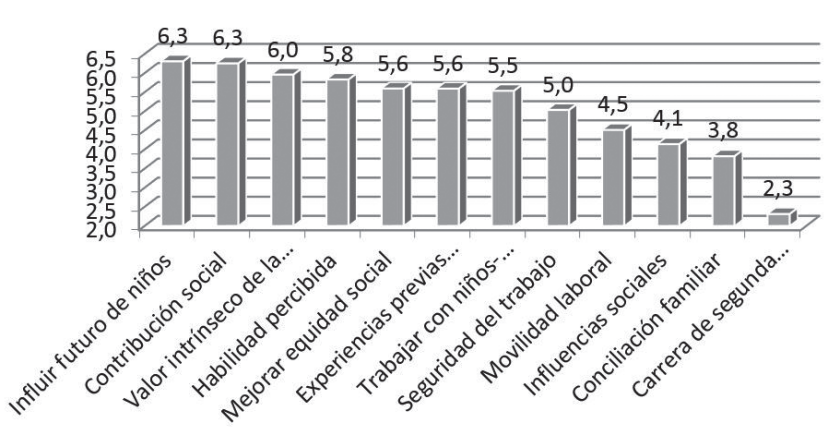

Fuente: elaboración propia.

\subsection{Importancia de los factores perceptuales}

Como se puede observar en la Figura 3, entre los factores perceptuales importantes para la elección de la carrera de Educación para el total de nuestros participantes están: la Satisfacción con la elección, en primer lugar; la Carrera exigente, en segundo lugar; y la Profesión exigente, en tercer lugar. La disuasión social y el salario no parecen ser muy importantes entre los factores perceptuales. 
Figura 3. Medias de las valoraciones de los factores perceptuales del FIT-Choice para el total

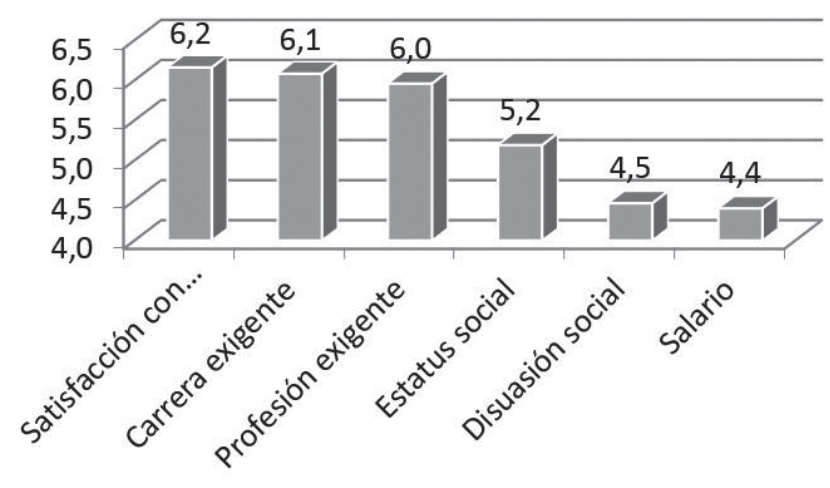

Fuente: elaboración propia.

\subsection{Factores para elección de ISFODOSU}

Además de las motivaciones y los factores perceptuales que contiene el FIT-Choice para determinar la elección de la carrera de Educación, nuestro cuestionario incluyó siete factores generales para elegir específicamente ISFODOSU como universidad:

1. Económico: costo, apoyos, becas.

2. Calidad: prestigio, excelencia.

3. Infraestructura: instalaciones, recursos, ubicación.

4. Administrativo: tamaño, requisitos, seguridad.

5. Programas: especialización, enfoque teórico-práctico.

6. Profesorado: conocimientos, experiencia.

7. Servicios: vida social, acciones comunitarias.

Los participantes debían evaluar los tres factores que consideraran más importantes. Debían asignarle un tres al factor más importante por el que ellos habían elegido ISFODOSU como universidad. Debían asignarle un dos al segundo factor en importancia y un uno al tercer factor en importancia. Durante el análisis hubo necesidad de eliminar las puntuaciones mayores a tres porque fueron dadas por error, pero solo fueron afectadas el 3\% de las respuestas.

La Figura 4 recoge las valoraciones del total de participantes para cada uno de los factores que influyeron en su elección de ISFODOSU como universidad. En esta figura podemos apreciar que el factor más decisivo fue la calidad de la universidad, seguido de cerca por el factor económico y luego por sus servicios. Los factores menos decisivos fueron la infraestructura, los programas y el profesorado.

Figura 4. Medias de las valoraciones de los factores que determinaron la elección de ISFODOSU por parte del total de participantes

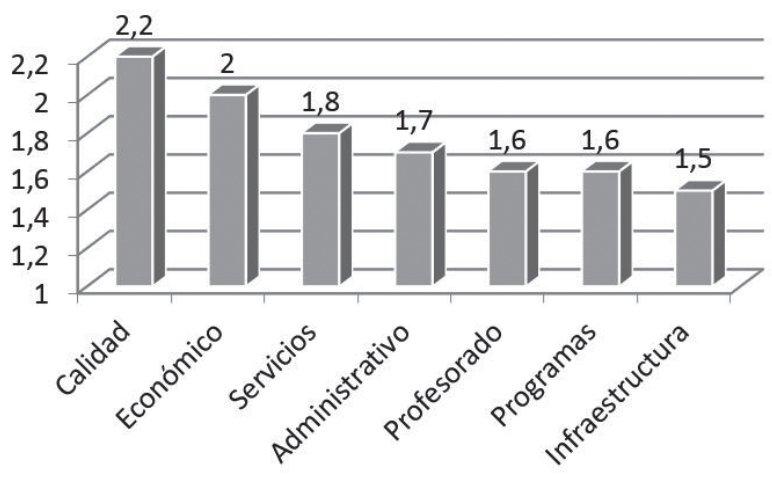

Fuente: elaboración propia.

\subsection{Recursos tecnológicos de ISFODOSU}

Para conocer la opinión de los participantes sobre los recursos tecnológicos (TIC) de ISFODOSU: computadoras, $\mathrm{Wi}-\mathrm{Fi}$, proyectores, pantallas, pizarras inteligentes, etc., nuestro cuestionario incluyó una pregunta sobre si la cantidad de los recursos tecnológicos de la universidad era adecuada y otra sobre si esos recursos tecnológicos estaban actualizados. Las respuestas se daban a través de una escala tipo Likert, donde cinco representaba el total acuerdo y uno representaba el total desacuerdo.

Para el total de participantes, las respuestas a ambas preguntas fueron muy positivas. La media de puntuación para la pregunta sobre la cantidad adecuada de TIC fue cercana a 4 (Media $=3.72, \mathrm{DE}=1.064) . \mathrm{Un} 45 \%$ estuvo de acuerdo y un $23 \%$ estuvo totalmente de acuerdo con la afirmación de que la cantidad de TIC de ISFODOSU era adecuada.

La pregunta sobre si los recursos TIC de ISFODOSU estaban actualizados recibió una reacción más favorable aún. La media de puntuación para esta pregunta fue todavía más cercana a cuatro (Media $=3.86$, $\mathrm{DE}=.989)$. Un $50 \%$ estuvo de acuerdo y un $25 \%$ estuvo totalmente de acuerdo con la afirmación de que los TIC de ISFODOSU estaban actualizados. 


\subsubsection{Diferencias en factores motivacionales según recinto}

Una vez confirmadas las propiedades psicométricas adecuadas de la escala FIT-Choice en sus factores motivacionales y perceptuales, pudimos analizar las puntuaciones de nuestros participantes en dichas escalas, de acuerdo con la influencia que pudieron haber ejercido sobre ellas nuestras variables independientes Recinto, Carrera y Sexo.

Al realizar el análisis de varianza (ANOVA, según sus siglas en inglés) con nuestras tres variables independientes, utilizando el procedimiento del modelo lineal general, encontramos que ocho de las motivaciones estuvieron influenciadas por el recinto en que estudiaban los participantes. Estas se pueden encontrar en la Tabla 8, junto con los resultados del ANOVA para el recinto.

Tabla 8. Resultados del ANOVA para las motivaciones que mostraron diferencias entre los recintos

\begin{tabular}{llllll}
\hline Fuente & gl & F & Sig. & f & $\mathbf{1}-\boldsymbol{\beta}$ \\
$\begin{array}{l}\text { Influir futuro de } \\
\text { niños }\end{array}$ & 5.706 & 5.025 & .000 & .72 & 1 \\
$\begin{array}{l}\text { Habilidad perci- } \\
\text { bida }\end{array}$ & 5.706 & 4.270 & .001 & .64 & 1 \\
$\begin{array}{l}\text { Mejorar equidad } \\
\text { social }\end{array}$ & 5.706 & 3.952 & .002 & .61 & 1 \\
$\begin{array}{l}\text { Valor intrínseco de } \\
\text { la carrera }\end{array}$ & 5.706 & 3.895 & .002 & .61 & 1 \\
$\begin{array}{l}\text { Influencias sociales } \\
5.706\end{array}$ & 3.525 & .004 & .56 & 1 \\
$\begin{array}{l}\text { Contribución } \\
\text { social }\end{array}$ & 5.706 & 3.186 & .007 & .53 & 1 \\
$\begin{array}{l}\text { Trabajar con ni- } \\
\text { nos-adolescentes }\end{array}$ & 5.706 & 2.756 & .018 & .48 & 1 \\
$\begin{array}{l}\text { Experiencias pre- } \\
\text { vias de enseńanza }\end{array}$ & 5.706 & 2.743 & .018 & .48 & 1 \\
\hline
\end{tabular}

Fuente: elaboración propia.
En esta tabla, las motivaciones están ordenadas de acuerdo con el tamaño del efecto, expresado en términos de $f$ (Cohen, 1992; Faul et al., 2012). Las motivaciones con mayor efecto en las diferencias entre recintos fueron Influir en el futuro de niños y adolescentes, Habilidad percibida para enseñar, Mejorar la equidad social y el Valor intrínseco de la carrera. Sin embargo, las demás fuentes también obtuvieron un tamańo del efecto grande.

En la Figura 5 podemos observar las valoraciones de la motivación Influir en el futuro de niños y adolescentes y sus diferencias entre los recintos. Con la ayuda de las comparaciones post hoc pudimos confirmar que los recintos que estuvieron en los primeros lugares en esta motivación fueron el LNNM, FEM y EMH, en donde esta motivación fue evaluada mucho más significativamente que en el UM.

Figura 5. Medias de las valoraciones de la motivación Influir en el futuro de los niños, según recinto

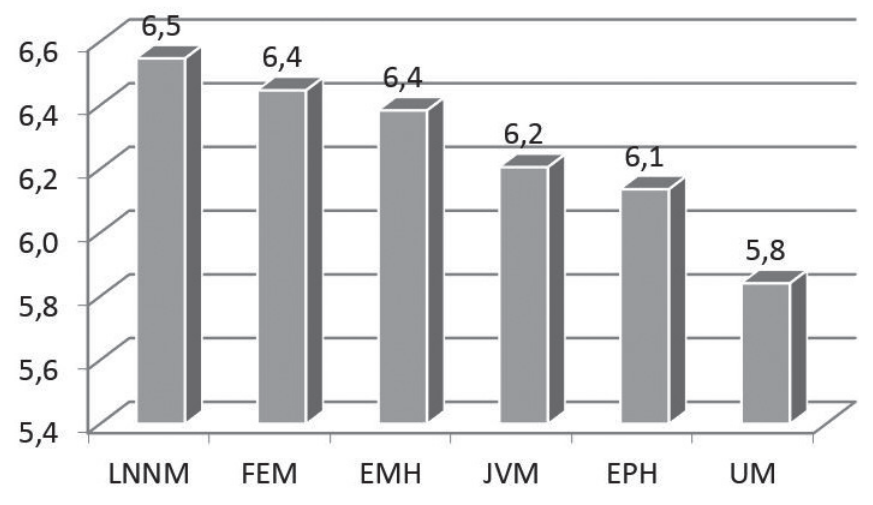

Fuente: elaboración propia.

Las medias de las valoraciones para la motivación Habilidad percibida para enseñar se muestran en la Figura 6. En esta motivación el LNNM quedó en primer lugar, pero los recintos en segundo (JVM y EMH) y tercer lugar (FEM) también quedaron significativamente por encima del UM. 
Figura 6. Medias de las valoraciones de la motivación Habilidad percibida, según recinto

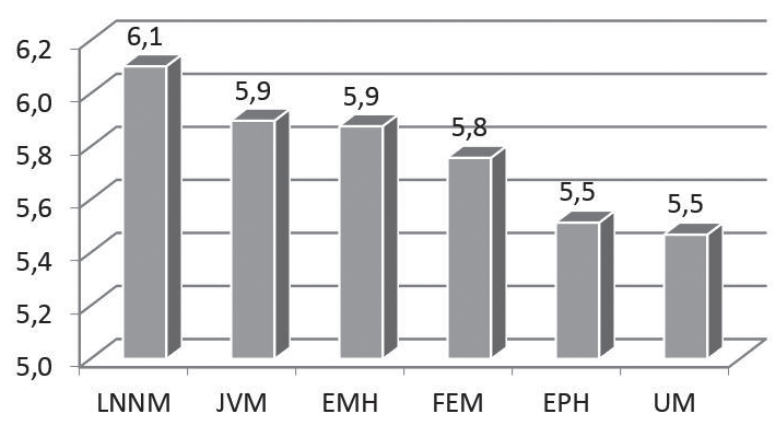

Fuente: elaboración propia.

En la Figura 7 se muestran las medias de las valoraciones para la motivación Mejorar la equidad social, entre los diferentes recintos. En esta figura se puede constatar que, aunque el FEM quedó en primer lugar, los recintos en segundo y tercer lugar (LNNM Y EPH) estuvieron todos significativamente más altos que el UM.

Figura 7. Medias de las valoraciones de la motivación Mejorar la equidad social, según recinto

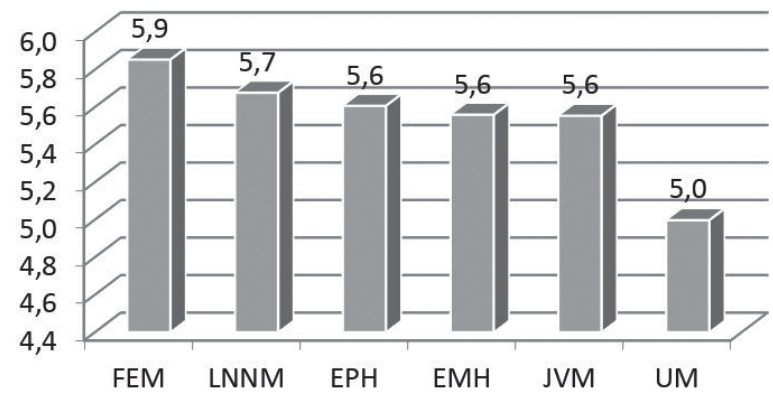

Fuente: elaboración propia.

Con el mismo tamaño del efecto que en la motivación de mejorar la equidad social, el Valor intrínseco de la carrera también mostró diferencias entre los recintos, las cuales se muestran en la Figura 8. Aquí se comprobó igualmente que, en los recintos que estaban en los primeros tres lugares, el LNNM, el JVM, el FEM y el EMH valorizaron esta motivación como significativamente más alta que en el UM.
Figura 8. Medias de las valoraciones de la motivación Valor intrínseco de la carrera, según recinto

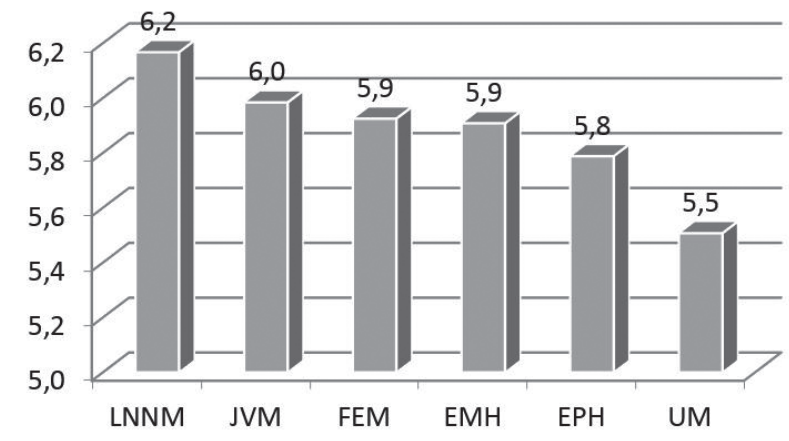

Fuente: elaboración propia.

Dentro de las motivaciones que mostraron diferencias entre los recintos, vemos en la Figura 9 las valoraciones que le otorgaron a la motivación Influencias sociales. En esta ocasión vemos dos grupos de recintos: aquellos que otorgaron mayor valor a las influencias sociales en su elección de carrera (LNNM, JVM y $\mathrm{EPH}$ ), frente a los recintos donde se otorgó muy poco valor a esta motivación (UM, FEM y EMH).

Figura 9. Medias de las valoraciones de la motivación Influencias sociales, según recinto

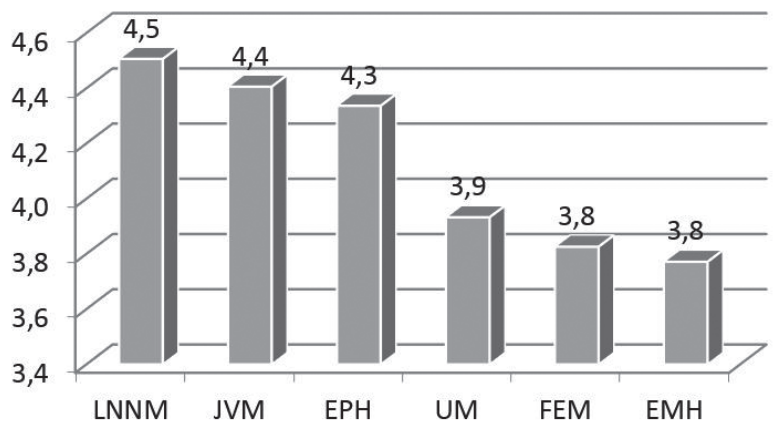

Fuente: elaboración propia.

Elegir la carrera de Educación para hacer una contribución social fue otra motivación que presentó diferencias entre los recintos, como se puede verificar en la Figura 10. En esta figura resalta en primer lugar el 
LNNM, pero los recintos que estuvieron en segundo y tercer lugar (EMH, FEN, JVM) también valorizaron esta motivación significativamente más alta que en el UM.

Figura 10. Medias de las valoraciones de la motivación Contribución social, según recinto

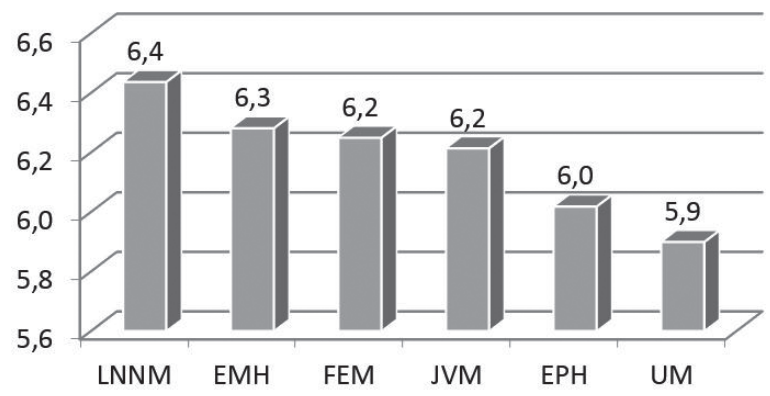

Fuente: elaboración propia.

Trabajar con niños y adolescentes fue otra de las motivaciones que presentaron diferencias significativas entre los recintos. En la Figura 11 podemos advertir que las diferencias se refieren a los dos extremos de la figura, el LNNM en el primer lugar y el UM en el último lugar. Los demás recintos se mantuvieron en una valoración promedio de esta motivación.

Figura 11. Medias de las valoraciones de la motivación Trabajar con niños y adolescentes, según recinto

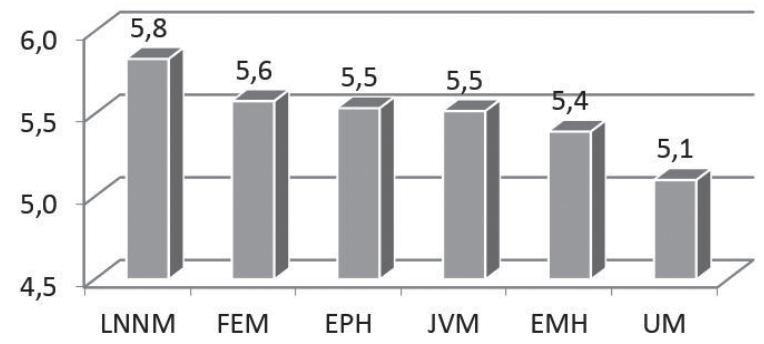

Fuente: elaboración propia.

La otra motivación que presentó diferencias entre los recintos fue Experiencias previas de enseñanza. En la Figura 12 vemos que las valoraciones de esta motivación fueron muy similares, excepto por los extremos de la figura, que mostraron diferencias significativas. En el LNNM, esta motivación fue significativamente más importante que en el UM.

Figura 12. Medias de las valoraciones de la motivación Experiencias previas de enseñanza, según recinto

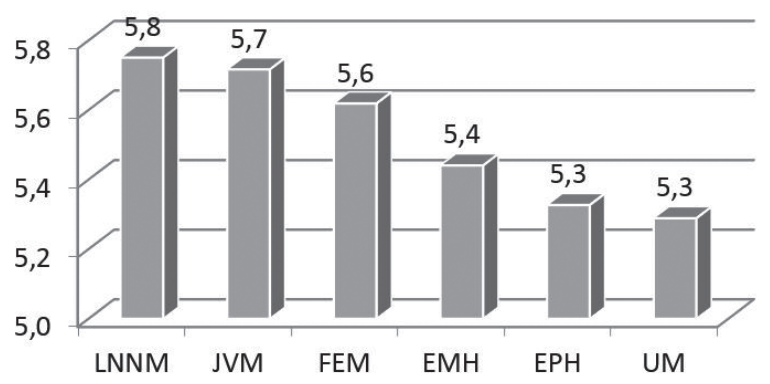

Fuente: elaboración propia.

\subsubsection{Diferencias en factores motivacionales según carrera}

En la Tabla 9 ofrecemos los detalles de la significación estadística de dos variables motivacionales que mostraron diferencias debido a las carreras que estudiaban los participantes: Seguridad del trabajo y Trabajar con niños-adolescentes.

Tabla 9. Resultados del ANOVA para las motivaciones que mostraron diferencias entre las carreras

\begin{tabular}{cccccc}
\hline Fuente & $g l$ & $F$ & Sig. & $f$ & $1-\beta$ \\
$\begin{array}{c}\text { Seguridad del } \\
\text { trabajo }\end{array}$ & 3.706 & 3.017 & .029 & .39 & 1 \\
$\begin{array}{c}\text { Trabajar con } \\
\text { niños-adoles- } \\
\text { centes }\end{array}$ & 3.706 & 3.310 & .02 & .40 & 1 \\
\hline
\end{tabular}

Fuente: elaboración propia.

En la Figura 13 podemos visualizar las diferencias de valoración para la motivación Seguridad del trabajo según las carreras que estudiaban los participantes. 
En esta figura se puede apreciar que los estudiantes de Educación Primaria y Secundaria tenían una valoración muy baja de la motivación Seguridad del trabajo, mientras que aquellos de Educación Inicial y Física le dieron una valoración moderada a esta motivación.

Figura 13: Medias de las valoraciones de la motivación Seguridad del trabajo, según carrera

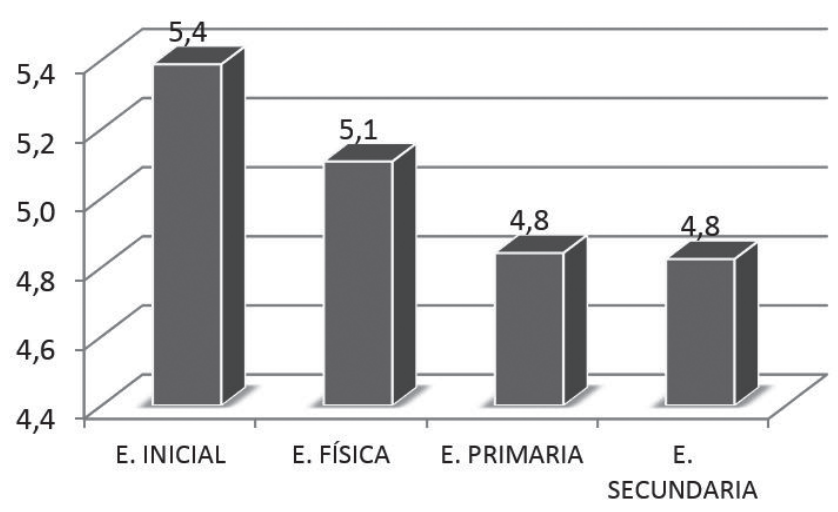

Fuente: elaboración propia.

En la Figura 14 podemos apreciar las diferencias entre las carreras con respecto a la motivación Trabajar con niños-adolescentes. Las participantes de la carrera de Educación Inicial tuvieron una valoración significativamente más alta de esta motivación, que la de aquellos que estudiaban las demás carreras.

Figura 14. Medias de las valoraciones de la motivación Trabajar con niños-adolescentes, según carrera

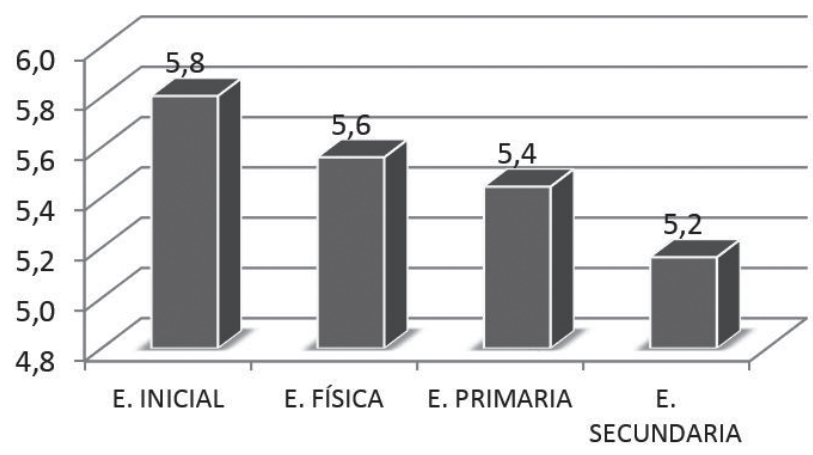

Fuente: elaboración propia.

\subsubsection{Diferencias en factores motivacionales según sexo}

En la Tabla 10 se pueden encontrar los resultados del ANOVA para las motivaciones que mostraron diferencias estadísticamente significativas entre los sexos.

Tabla 10. Resultados del ANOVA para las motivaciones que mostraron diferencias entre los sexos

\begin{tabular}{|c|c|c|c|c|c|}
\hline Fuente & gl & $\mathbf{F}$ & Sig. & f & $1-\beta$ \\
\hline $\begin{array}{l}\text { Trabajar con } \\
\text { niños-adolescentes }\end{array}$ & 1.706 & 34.181 & .000 & .92 & 1 \\
\hline $\begin{array}{l}\text { Valor intrínseco de } \\
\text { la carrera }\end{array}$ & 1.706 & 19.340 & .000 & .61 & 1 \\
\hline Habilidad percibida & 1.706 & 17.265 & .000 & .56 & 1 \\
\hline Contribución social & 1.706 & 17.253 & .000 & .56 & 1 \\
\hline $\begin{array}{l}\text { Influir futuro de } \\
\text { niños }\end{array}$ & 1.706 & 10.783 & .001 & .42 & 1 \\
\hline $\begin{array}{l}\text { Experiencias previas } \\
\text { de enseñanza }\end{array}$ & 1.706 & 8.512 & .004 & .37 & 1 \\
\hline $\begin{array}{l}\text { Mejorar equidad } \\
\text { social }\end{array}$ & 1.706 & 6.210 & .013 & .10 & .72 \\
\hline
\end{tabular}

Fuente: elaboración propia.

Esta tabla también está ordenada de mayor a menor según el tamaño del efecto en términos de $f$. En otras palabras, las mayores diferencias entre hombres y mujeres estuvieron en torno a las motivaciones Trabajar con niños-adolescentes, Valor intrínseco de la carrera, Habilidad percibida y Contribución social. La importancia de la diferencia entre sexos fue grande también en las motivaciones Experiencias previas de enseñanza e Influir en el futuro de niños y adolescentes. La motivación en la cual hubo una mínima diferencia entre hombres y mujeres fue Mejorar la equidad social. 
En la Figura 15 podemos apreciar las diferencias entre las medias de los hombres y las mujeres participantes en las motivaciones que mostraron diferencias significativas entre los sexos.

Figura 15. Medias de las valoraciones de las motivaciones que mostraron diferencias significativas entre los sexos

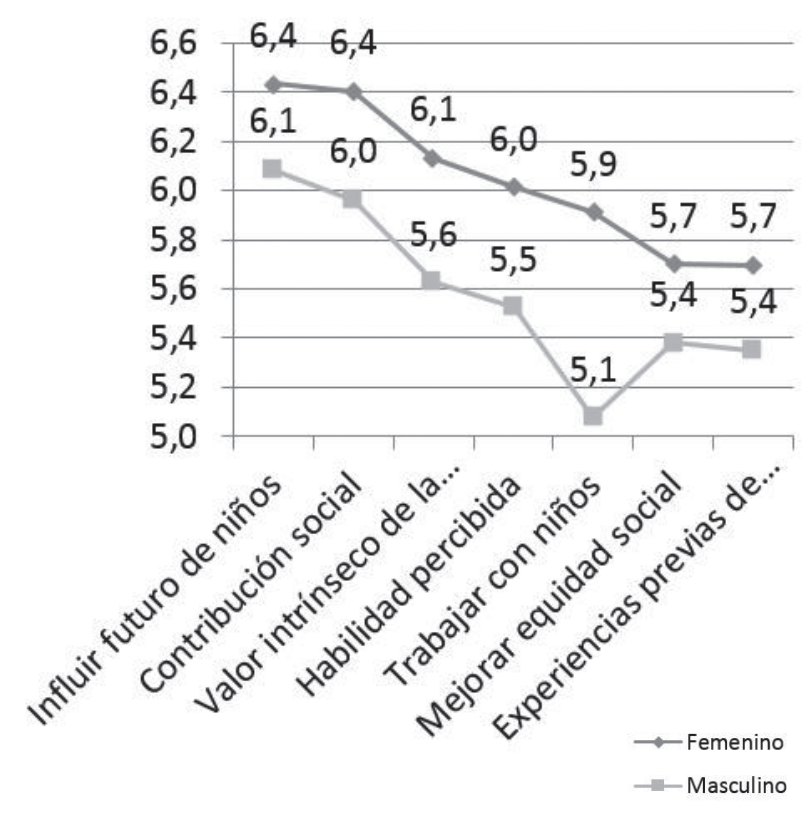

Fuente: elaboración propia.

En esta figura se nota que en todas estas motivaciones las mujeres mostraron una valoración mucho más alta que los hombres, especialmente en Trabajar con niños y adolescentes, la cual fue escasamente valorizada por los hombres.

\subsubsection{Diferencias en factores perceptuales según recinto}

$\mathrm{Al}$ realizar los ANOVA con nuestras tres variables independientes utilizando el modelo lineal general, encontramos que cinco de los factores perceptuales del FIT-Choice estuvieron influenciados por el recinto en que estudiaban los participantes. Estos factores se encuentran en la Tabla 11, junto con los resultados del ANOVA para el recinto.
Tabla 11. Resultados del ANOVA para factores perceptuales que mostraron diferencias entre los recintos

\begin{tabular}{cccccc}
\hline Fuente & $\mathbf{g l}$ & $\mathbf{F}$ & Sig. & $\mathbf{f}$ & $\mathbf{1}-\boldsymbol{\beta}$ \\
Estatus social & 5.692 & 6.090 & .000 & .21 & .996 \\
$\begin{array}{c}\text { Satisfacción con } \\
\text { elección }\end{array}$ & 5.692 & 5.127 & .000 & .19 & .987 \\
$\begin{array}{c}\text { Profesión exigente } \\
\text { Carrera exigente }\end{array}$ & 5.692 & 4.595 & .000 & .18 & .975 \\
Disuasión social & 5.692 & 4.094 & .001 & .17 & .959 \\
\hline
\end{tabular}

Fuente: elaboración propia.

Entre los factores perceptuales del FIT-Choice que mostraron diferencias entre los recintos de ISFODOSU, el más importante fue el Estatus social, seguido de Satisfacción con la elección. El que menos importancia tuvo para estas diferencias fue el de Disuasión social.

En la Figura 16 podemos ver las diferencias que se encontraron en el factor perceptual Estatus social entre los recintos. En esta figura podemos observar que el LNNM y el EMH tuvieron las mejores evaluaciones en este factor, elevándose significativamente frente a los recintos UM, FEM y EPH.

Figura 16. Medias de las valoraciones para el factor perceptual Estatus social, según recinto

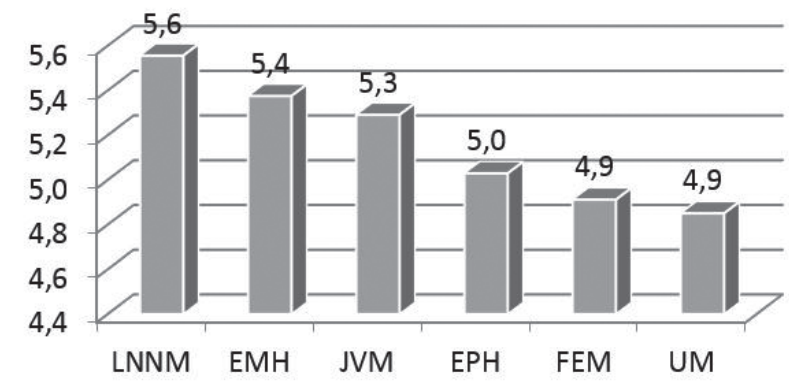

Fuente: elaboración propia.

El factor perceptual del FIT-Choice Satisfacción con la elección también presentó diferencias significativas entre las valoraciones hechas por los participantes de los diferentes recintos de la universidad. De nuevo, el LNNM, junto al FEM en segundo lugar, 
produjeron las evaluaciones más altas en este factor, comparadas con las bajas evaluaciones hechas por los participantes del UM y EPH. En la figura 17 podemos visualizar estas diferencias.

Figura 17. Medias de las valoraciones para el factor perceptual Satisfacción con la elección, según recinto

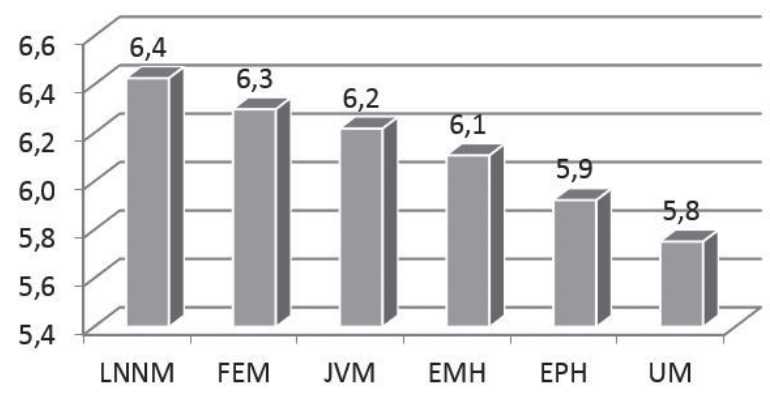

Fuente: elaboración propia.

El tercer lugar en importancia, entre los factores perceptuales que mostraron diferencias entre los recintos, fue para el factor Profesión exigente. En la Figura 18 podemos verificar que el LNNM y el FEM obtuvieron las medias de valoración más altas de este factor, significativamente por encima de la media de UM. Los demás recintos se encontraron en un nivel promedio.

Figura 18. Medias de las valoraciones para el factor perceptual Profesión exigente, según recinto

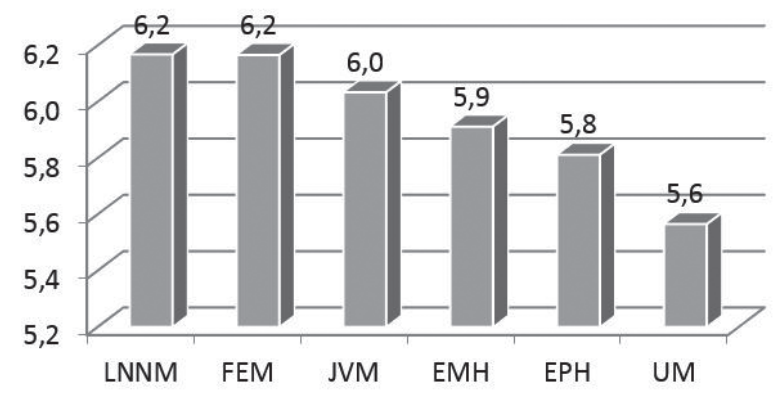

Fuente: elaboración propia.

La Carrera exigente fue otro de los factores perceptuales del FIT-Choice que mostró diferencias en sus valoraciones debido a los distintos recintos. En la
Figura 19 podemos ver que el FEM y el LNNM se destacan por sus altas evaluaciones de este factor, sobre todo del extremo inferior de la figura, donde se encuentran las medias de valoraciones del EPH y el UM, significativamente más bajas.

Figura 19. Medias de las valoraciones para el factor perceptual Carrera exigente, según recinto

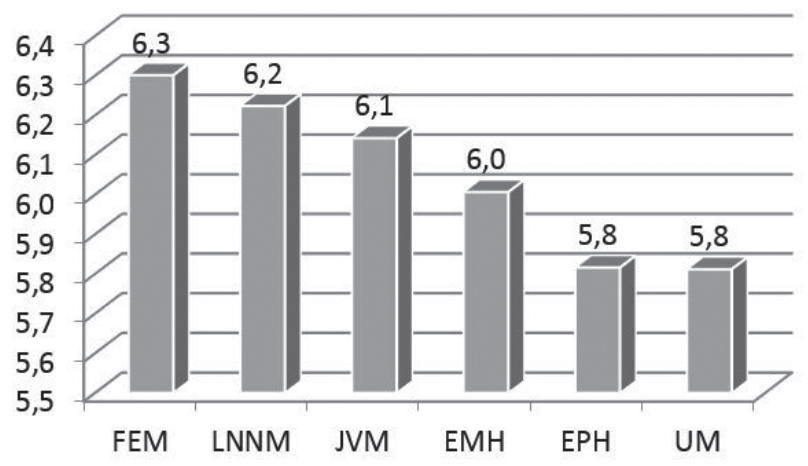

Fuente: elaboración propia.

La Disuasión social fue el factor menos importante entre aquellos factores perceptuales del FIT-Choice que mostraron diferencias debido al recinto. En la Figura 20 podemos comprobar que, de nuevo, el LNNM obtuvo el primer lugar en las evaluaciones de este factor, seguido de cerca por las evaluaciones del EPH y el EMH, todas significativamente más altas que las evaluaciones del JVM.

Figura 20. Medias de las valoraciones para el factor perceptual Disuasión social, según recinto

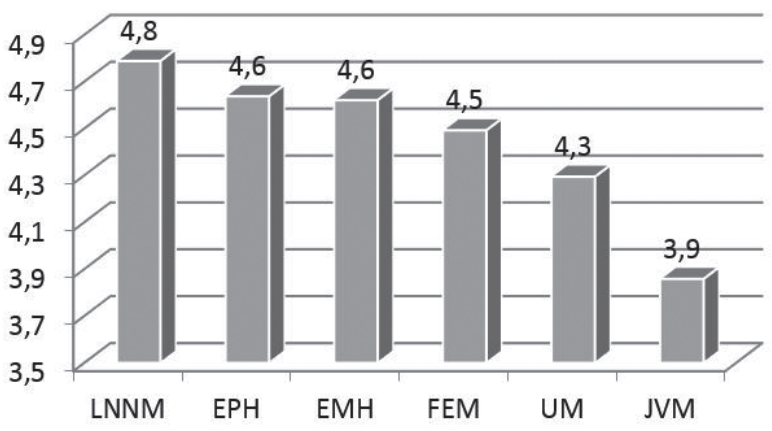

Fuente: elaboración propia. 


\subsubsection{Diferencias en factores perceptuales según sexo}

En los factores perceptuales del FIT-Choice no encontramos ninguna diferencia entre las evaluaciones de las participantes debido a las carreras que cursaban, pero sí entre las valoraciones que hacían los diferentes sexos. En la Tabla 12 se encuentran los resultados del ANOVA para los factores perceptuales que mostraron diferencias significativas entre los sexos.

Tabla 12. Resultados del ANOVA para factores perceptuales que mostraron diferencias entre los sexos

\begin{tabular}{|c|c|c|c|c|c|}
\hline Fuente & gl & $\mathbf{F}$ & Sig. & f & $1-\beta$ \\
\hline $\begin{array}{l}\text { Carrera } \\
\text { exigente }\end{array}$ & 1.706 & 9.806 & .002 & .12 & .889 \\
\hline $\begin{array}{l}\text { Satisfacción } \\
\text { con elección }\end{array}$ & 1.706 & 6.693 & .010 & .10 & .765 \\
\hline $\begin{array}{c}\text { Disuasión } \\
\text { social }\end{array}$ & 1.706 & 4.068 & .044 & .08 & .546 \\
\hline
\end{tabular}

Fuente: elaboración propia.

El factor perceptual que provocó más diferencia fue Carrera exigente y el que menos efecto tuvo fue Disuasión social, pero todos los tamaños del efecto fueron pequeños. En la Figura 21 se pueden observar las diferencias entre los sexos en estos tres factores perceptuales. Las mujeres dieron una valoración más alta que los hombres en todos ellos. La Disuasión social parece el menos importante de todos.
Figura 21. Medias de las valoraciones de factores perceptuales del FIT-Choice que mostraron diferencias significativas entre los sexos

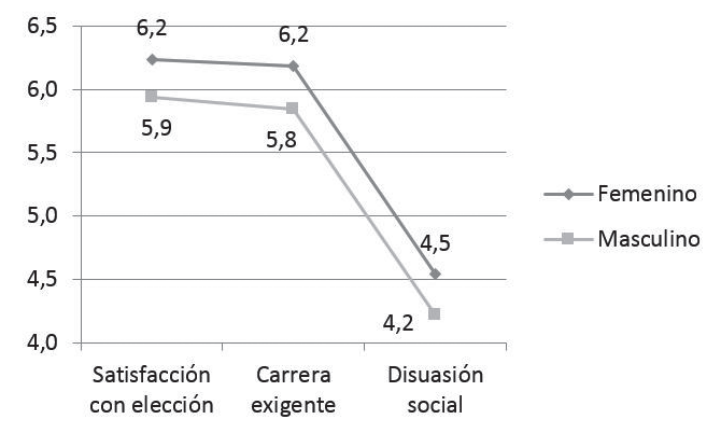

Fuente: elaboración propia.

\subsubsection{Diferencias entre las variables indepen- dientes para elegir ISFODOSU}

Como teníamos siete variables dependientes por analizar, para claridad de presentación e interpretación, en lugar de un solo ANOVA de tres factores, realizamos dos ANOVA de una vía para nuestras variables independientes Recinto y Carrera, cada una, así como Pruebas $t$ para muestras independientes para la variable independiente Sexo.

Según los resultados del ANOVA de una vía para determinar las diferencias entre los recintos con respecto a los factores que determinaron su elección de ISFODOSU como universidad, no apareció ninguna diferencia significativa entre recintos, en ninguno de los factores de elección. La importancia de los factores siguió siendo la misma del total en todos los recintos.

Por otra parte, tampoco aparecieron diferencias significativas en los resultados del ANOVA hecho para determinar si aparecían diferencias entre las carreras. La importancia de los factores siguió siendo la misma del total en todas las carreras.

Con respecto a la variable Sexo, solo dos factores mostraron diferencias debido al sexo de los participantes; por un lado, el factor económico, $t(620)=3.86$, $p=.000$, con un tamaño del efecto bastante grande $(d=.32)$ y una potencia de la prueba muy alta $(1-\beta=.97)$; y por otro lado, el factor de servicios, $t$ $(150)=-2.729, p=.007$, con un tamaño del efecto 
también bastante grande $(d=.44)$, y una potencia de la prueba alta $(1-\beta=.76)$.

Estas diferencias entre los sexos se resumen en la Figura 22. Aquí podemos ver ambos sexos en franca interacción. Mientras para los hombres el factor económico fue más decisivo que el factor de servicios para su elección de ISFODOSU como universidad, para las mujeres fue más decisivo el factor de servicios que el económico. En los demás factores no hubo diferencias con respecto a la valoración del total (Figura 4).

Figura 22. Medias de las valoraciones de los factores que determinaron diferencias entre los sexos para la elección de ISFODOSU

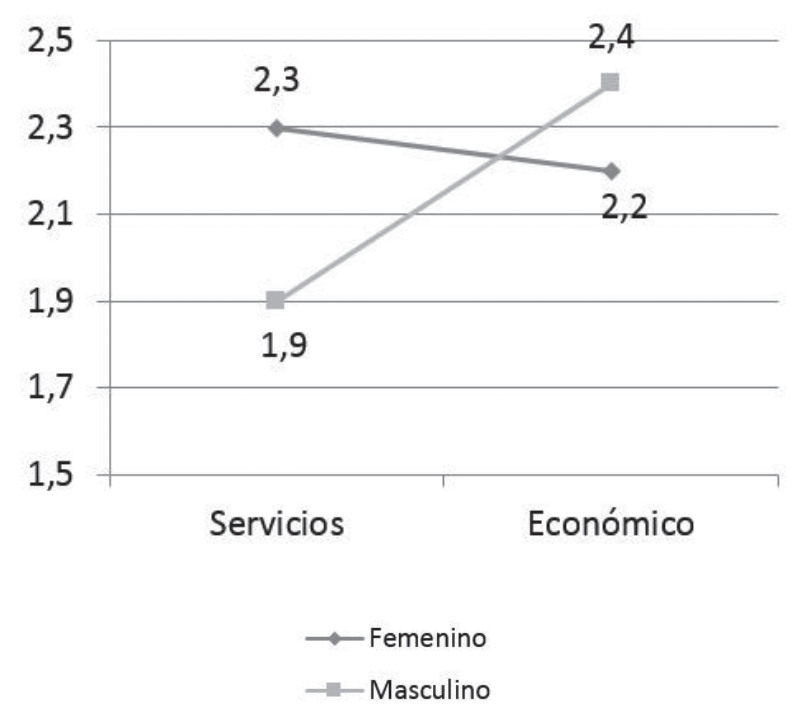

Fuente: elaboración propia.

\subsubsection{Diferencias entre los recintos sobre las TIC en ISFODOSU}

Para ambas preguntas se realizaron ANOVAS de tres factores con nuestras variables independientes. El ANOVA para determinar las diferencias de opinión sobre si la cantidad de TIC era adecuada mostró que ni la carrera, ni el sexo de los participantes mostraron diferencias en sus valoraciones sobre las TIC. Sin embargo, entre los recintos sí apareció una diferencia significativa, $F(5.711)=4.826, p=.000$, con un tamańo pequeńo del efecto $(f=.18)$, y una potencia muy alta $(1-\beta=.98)$.

Esta diferencia se puede visualizar en la Figura 23, donde podemos ver que la mejor opinión sobre la cantidad adecuada de TIC en ISFODOSU apareció en el JVM, encontrándose significativamente por encima de los demás recintos. La mayoría de los demás recintos tuvo una opinión cercana al promedio, pero el UM mostró una opinión significativamente más baja que las de todos los demás.

Figura 23. Medias de acuerdo con la afirmación de que la cantidad de los recursos TIC de ISFODOSU era adecuada, según recinto

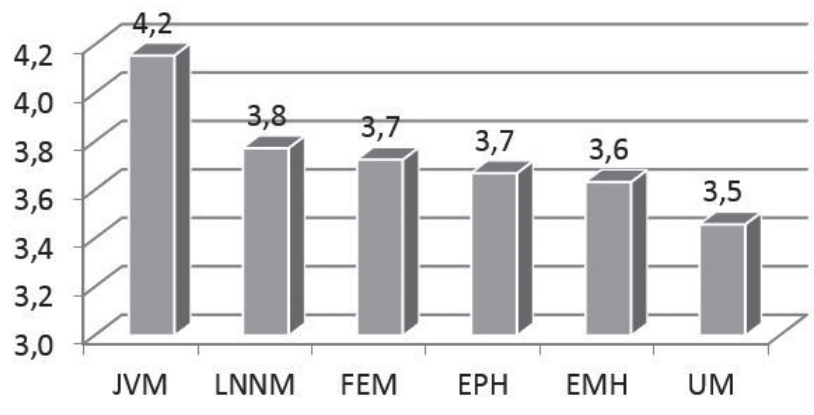

Fuente: elaboración propia.

El ANOVA realizado para determinar la influencia de los recintos, las carreras y el sexo de los participantes sobre su opinión acerca de la actualización de los recursos TIC de ISFODOSU mostró diferencias significativas entre los recintos, $F(5,708)=5.614$, $p=.000$, con un tamaño pequeño del efecto $(f=.038)$ y una potencia muy alta $(1-\beta=.993)$; así como entre los sexos, $F(1,708)=9.456, p=.002$, con un tamaño muy pequeño del efecto $(f=.04)$ y una potencia baja $(1-\beta=.22)$.

La Figura 24 recoge las diferencias entre los recintos. En ella podemos distinguir claramente dos grupos de recintos. Aquellos que tuvieron una actitud favorable, cerca de cuatro en promedio, hacia la actualización de los TIC en ISFODOSU: JVM, EPH, LNNM y FEM, así como los dos recintos cuya opinión fue significativamente inferior a la de los demás: EMH y UM. 
Figura 24. Medias de acuerdo con la afirmación de que los recursos TIC de ISFODOSU estaban actualizados, según recinto

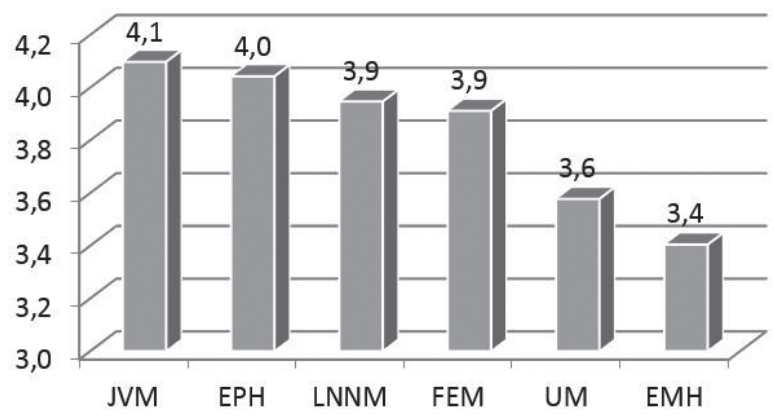

Fuente: elaboración propia.

\subsubsection{Diferencias entre los sexos sobre la actua- lización de los TIC en ISFODOSU}

De acuerdo con el ANOVA recién mencionado, en general, las mujeres evaluaron los recursos TIC de ISFODOSU como más actualizados (Media $=4$, $\mathrm{DE}=.049)$ que los hombres (Media $=3.7, \mathrm{DE}=.071)$.

\section{Discusión y conclusiones}

\subsection{Cualidades psicométricas de la escala FIT-Choice}

Era necesario confirmar las cualidades psicométricas de la escala FIT-Choice utilizada para determinar los factores que influyeron en la elección de los estudios de Educación por parte de nuestros participantes, debido a que solo existe un estudio en el país que ha usado esta escala y fue realizado en otro contexto (Tomás, 2014).

La bondad de ajuste de los modelos de factores motivacionales y perceptuales de esta escala fueron confirmadas, al igual que en otros estudios similares ya citados. Las cargas factoriales de los ítems fueron también todas confirmadas, así como los índices de confiabilidad y las correlaciones entre los factores.

Esto implica que este estudio aporta un instrumento de carácter internacional para poder comparar las motivaciones de nuestros estudiantes con las de todos los países que han validado esta escala. Además, nos coloca en posición de poder elaborar normas para poder utilizar esta escala como un instrumento predictivo para determinar la propiedad de las motivaciones para estudiar Educación.

Al respecto, recomendamos a la universidad que mantenga las mediciones de las motivaciones de los estudiantes para, en un futuro, correlacionarlas con el rendimiento escolar. De esta manera, la escala FIT-Choice podría servirnos para predecir también el éxito en los estudios pedagógicos.

\subsection{Importancia relativa de los factores motiva- cionales y perceptuales de la escala FIT-Choice}

\section{Motivaciones}

El factor de segundo orden más importante para nuestros aspirantes a docentes fue el valor de utilidad social. Este se considera de segundo orden porque incluye varios factores motivacionales relacionados. Entre estos el más determinante para la elección de la carrera de Educación fue Influir en el futuro de niños y adolescentes; es decir, nuestros estudiantes, en general, escogieron su carrera por el afán de formar las generaciones futuras. Esta motivación fue encontrada en un lugar de menor importancia en el estudio de Tomás (2014).

También tienen alta utilidad social Hacer una contribución social, devolver en servicio lo recibido por la sociedad; Trabajar con niños y adolescentes; y Mejorar la equidad social trabajando a favor de los socialmente desfavorecidos.

Por otra parte, como parte del valor intrínseco de la carrera, se encuentra el interés por la enseñanza, el gusto por enseñar, el cual está íntimamente relacionado con la autopercepción de las habilidades percibidas para enseñar, también motivaciones muy importantes para nuestros participantes.

En el otro extremo de la balanza encontramos el valor de utilidad social, también un factor de segundo orden, porque incluye las motivaciones de poca importancia para elegir Educación como carrera, como tratar de complacer a la familia (Conciliación familiar), aspirar a puestos más altos (Movilidad social) y la Seguridad en el trabajo. 
Por último, encontramos que la carrera de Educación no fue la última opción que tuvieron nuestros participantes. La mayoría tenía claro que quería estudiar esta carrera.

\section{Percepciones}

Entre los factores perceptuales que influyeron en la elección de nuestros participantes, el más importante fue la Satisfacción con la elección tomada como una decisión seria, en un lugar más alto que los factores perceptuales de la exigencia de la profesión encontrados por Tomás (2014), como la Carrera exigente, por su conocimiento técnico especializado, y la Profesión exigente, por su carga de trabajo y esfuerzo emocional.

El factor perceptual menos importante para nuestros participantes fue, tal y como encontró Tomás (2014), el salario, parte del retorno de la profesión, aunque en nuestro estudio tampoco tuvo mucha importancia la disuasión social para escoger carreras alternativas. Parece claro que nuestros estudiantes no escogieron su carrera para hacerse ricos o por desánimo.

\subsection{Factores y recursos tecnológicos que influ- yeron en la elección de ISFODOSU}

En la elección de ISFODOSU como universidad influyó primeramente la calidad, su prestigio y su excelencia académica. Esto quiere decir que la universidad se encuentra bien posicionada entre un blanco de público que la percibe como la institución de referencia en la Enseñanza pedagógica superior en el país, incluso por encima del factor económico. Este último factor fue, naturalmente, también muy importante, puesto que la universidad cuenta con asistencia estatal, becas y ayuda económica que la convierten en una opción muy favorecida.

Favoreció también la elección de ISFODOSU la imagen que tenían los participantes sobre sus recursos tecnológicos. La imagen que tenía la gran mayoría de los participantes sobre las TIC de la universidad era que estaban adecuadas en su cantidad y además actualizadas.

\subsection{Diferencias entre recintos}

Las diferencias entre los recintos se centraron en las motivaciones, las percepciones y las opiniones sobre las TIC de ISFODOSU. No aparecieron diferencias entre los recintos con relación a los factores importantes para elegir la universidad.

Con respecto a las motivaciones, el recinto que mostró las más altas valoraciones fue el LNNM, excepto en la búsqueda de Equidad social, en la cual lo encontramos en el segundo lugar. En general, el FEM y el JVM se posicionaron en el segundo lugar, y el EMH y el EPH en tercer lugar. El UM ocupó sistemáticamente el último lugar en las valoraciones de las motivaciones, excepto en las Influencias sociales, en las cuales ocupó un tercer lugar.

En cuanto a los factores perceptuales del FIT-Choice, encontramos casi los mismos rangos que en las motivaciones. De nuevo el LNNM mostró las mejores valoraciones, excepto en las de la Carrera exigente, en la cual apareció en segundo lugar. En este caso, quienes se encontraron en segundo lugar fueron el FEM y el EMH, estando en tercer lugar el JVM y el EPH. Otra vez el UM apareció en último lugar, excepto en el factor perceptual Disuasión social, donde el último lugar lo ocupó el JVM.

El orden de los recintos fue diferente en torno a la opinión sobre los TIC de la universidad. Esta vez quien ocupó el primer lugar en opinión fue el JVM, seguido por el LNNM y el EPH, y luego por el FEM y el UM. Quien ocupó aquí el último lugar fue el EMH.

Recomendamos a la universidad tomar acciones de cambio de actitudes para mejorar las motivaciones y las percepciones, especialmente del UM, el EPH y el JVM. Con respecto a las opiniones sobre las TIC, sería necesario revisar la dotación de los recintos EMH, UM y FEM para mejorarlas.

\subsection{Diferencias entre carreras}

Hablando de las carreras, las diferencias fueron muy pocas y se ciñeron a dos motivaciones. En las motivaciones sobre Seguridad del trabajo y Trabajar con niños y adolescentes siempre estuvo en primer 
lugar la Educación Inicial; en segundo, la Educación Física; en tercero, la Educación Primaria; y, en último, la Educación Secundaria.

\subsection{Diferencias entre sexos}

El sexo de los participantes produjo diferencias entre las motivaciones más importantes y siempre fueron favorables a las mujeres. No en balde las mujeres eran mayoría en la universidad, pues se mostraron mejor motivadas que los hombres, especialmente en la motivación de Trabajar con niños y adolescentes. Con respecto a los factores perceptuales ocurrió lo mismo. Las mujeres les dieron valoraciones más altas que los hombres.

Entre los factores importantes para elegir ISFODOSU como universidad, encontramos que los hombres dieron mayor importancia que las mujeres al factor económico, mientras que las mujeres les dieron más importancia que los hombres a los servicios de la universidad. Esto podría ser explicado por un elemento cultural que otorga una mayor habilidad social a las mujeres.

Por último, no encontramos diferencias entre los sexos con respecto a la opinión sobre lo adecuado de la cantidad de TIC en la universidad, pero sí con respecto a su actualización, pues aquí la opinión de las mujeres fue más favorable que la de los hombres.

\subsection{Importancia de la validación de la escala FIT-Choice en el país para los estudiantes de Peda- gogía}

En general, podemos afirmar que el impacto de nuestros resultados de investigación fue alto, dado que corroboran hallazgos y resultados similares de estudios anteriores.

En nuestro país, este trabajo resulta original y relevante, y se destaca por presentar una muestra representativa y un diseño de investigación óptimo. Abordar las características de entrada de los estudiantes de magisterio supone una contribución de interés que ayudará a un mejor conocimiento de la profesión docente y, al mismo tiempo, permitirá una formación de maestros con un diseño de los planes de estudio más ajustado. La investigación presentada, por tanto, está llamada a tener un impacto en la comunidad científica y en la práctica de aula.

Reconocimiento: la realización de este trabajo contó con el apoyo financiero de ISFODOSU.

\section{Referencias}

Ávalos, B., Cavada, P., Pardo, M. \& Sotomayor, C. (2010). La profesión docente: temas y discusiones en la literatura internacional. Estudios pedagógicos (Valdivia), 36(1), 235-263. Doi: https://doi. org/10.4067/s0718-07052010000100013

Berger, J. \& D’Ascoli, Y. (2012). Motivations to become vocational education and training educators: a person-oriented approach. Vocations and Learning, 5(3), 225-249. Doi: https://doi. org/10.1007/s12186-012-9075-z

Bergmark, U., Lundström, S., Manderstedt, L. \& Palo, A. (2018). Why become a teacher? Student teachers' perceptions of the teaching profession and motives for career choice. European Journal of Teacher Education, 41(3), 266-281. Doi: https://doi.org/10.1080/02619768.2018.144 8784

Bruinsma, M. \& Jansen, E. (2010). Is the motivation to become a teacher related to pre-service teachers' intentions to remain in the profession? European Journal of Teacher Education, 33(2), 185-200. Doi: https://doi.org/10.1080/02619760903512927

Calvo, G. (2006). La profesionalización docente en Colombia. Documento presentado por la Fundación Compartir en la mesa de trabajo del Nuevo Plan Decenal de Educación, 2015. https://bit.ly/ 2Nm7XPG

Cervini, R., Dari, N. \& Quiroz, S. (2014). Estructura familiar y rendimiento académico en países de América Latina. Los datos del Segundo Estudio Regional Comparativo y Explicativo. Revista Mexicana de Investigación Educativa, 19(61), 569-597. Doi: https://doi.org/10.22201/iisue. 24486167e.2016.151.54884

Cohen, J. (1992). A power primer. Psychological bulletin, 112(1), 155. Doi: https://doi.org/ 10.1037/0033-2909.112.1.155 
Creemers, B. \& Kyriakides, L. (2007). The dynamics of educational effectiveness: A contribution to policy, practice and theory in contemporary schools. London: Routledge. Doi: https://doi.org/10. 4324/9780203939185

Creemers, B., Kyriakides, L. \& Sammons, P. (2010). Methodological advances in educational effectiveness research. London: Routledge. Doi: https:// doi.org/10.4324/9780203851005

Elacqua, G., Hincapíe, D., Vegas, E. \& Alfonso, M. (2018). Profesión: Profesor en América Latina ¿Por qué se perdió el prestigio docente y cómo recuperarlo? Banco Interamericano de Desarrollo. Doi: https://doi.org/10.18235/0001172

Eren, A. \& Tezel, K. (2010). Factors influencing teaching choice, professional plans about teaching, and future time perspective: A mediational analysis. Teaching and Teacher Education, 26(7), 1416-1428. Doi: https://doi.org/10.1016/j. tate.2010.05.001

Faul, F., Erdfelder, E., Buchner, A. \& Lang, A. (2012). G* POWER (Version 3.1. 5.) [Computer Program]. Germany: Universitat Kiel. https:// bit.ly/2TvGsqT

Fokkens-Bruinsma, M. \& Canrinus, E. (2012) The Factors Influencing Teaching (FIT) Choice scale in a Dutch teacher education program. Asia-Pacific Journal of Teacher Education, 40(3), 249-269. Doi: https://doi.org/10.1080/ 1359866X.2012.700043.

Frei, A., Berweger, S. \& Buschor, C. (2017). Men considering (and choosing) teaching as a career: what accounts for their decision to become a teacher? European Journal of Teacher Education, 40(4), 535-549. Doi: https://doi.org/10.1080/ 02619768.2017 .1315397

García-Poyato, J. \& Cordero, A. (2019). La profesión docente en crisis: disminución de la matrícula normalista en México. Archivos Analíticos de Politicas Educativas, 27(103). Doi: https://doi. org/10.14507/epaa.27.4625

Gratacós, G. \& López-Jurado, M. (2016). Validación de la versión en español de la escala de los factores que influyen en la elección de los estudios de educación (FIT-choice). Revista de Educación, (372), 87-105. Doi: https://doi.org/10.4438/1988592X-RE-2015-372-316.
Guerriero, G., \& Deligiannidi, K. (2017). The teaching profession and its knowledge base. In S. Guerriero (Ed.). Pedagogical Knowledge and the Changing Nature of the Teaching Profession (19-35). OECD. Doi: https://doi.org/10. $1787 / 9789264270695-3-e n$

Han, J. \& Yin, H. (2016). Teacher motivation: Definition, research development and implications for teachers. Cogent Education, 3(1), 1217819. Doi: https://doi.org/10.1080/2331186X.2016. 1217819

Hanushek, E. (2016). What matters for student achievement. Education Next, 16(2), 18-26. https://bit.ly/35ttrAa

Jugoviæ, I., Marušiæ, I., Ivanec, T. \& Vidoviæ, V. (2012). Motivation and personality of preservice teachers in Croatia. Asia-Pacific Journal of TeacherEducation, 40(3),271-287.Doi:https:// doi.org/10.1080/1359866x.2012.700044

Klassen, R., Al-Dhafri, S., Hannok, W. \& Betts, S. (2011). Investigating pre-service teacher motivation across cultures using the Teachers' Ten Statements Test. Teaching and Teacher Education, 27(3), 579-588. Doi: https://doi.org/10. $1016 /$ j.tate. 2010.10 .012

Kyriakides, L., Creemers, B. \& Charalambous, E. (2019). Searching for differential teacher and school effectiveness in terms of student socioeconomic status and gender: Implications for promoting equity. School Effectiveness and School Improvement, 30(3), 286-308. Doi: https://doi. org/10.1080/09243453.2018.1511603

Lin, E., Shi, Q., Wang, J., Zhang, S. \& Hui, L. (2012). Initial motivations for teaching: comparison between preservice teachers in the Unites States and China. Asia-Pacific Journal of Teacher Education, 40(3), 227-248. Doi: https://doi. org/10.1080/1359866x.2012.700047

Martínez-de-la-Hidalga, Z. \& Villardón-Gallego, L. (2018). El prestigio social de la profesión según los futuros docentes de Educación Primaria y Secundaria. Profesorado. Revista de Curriculum y Formación de Profesorado, 22(2), 289-308. Doi: https://doi.org/10.30827/profesorado. v22i 2.7724 
Melhuish, E., Sammons, P., Sylva, K., Siraj-Blatchford, I. \& Taggart, B. (2014). Effective Pre-School and Primary Education 3-11 Project. London: University of London. Doi: https://doi.org/10.14507/ epaa.v22.1893

Patrinos, H. (2007). Factores determinantes del aprendizaje y calidad de la educación en México. En Miranda, F., Patrinos, H. \& López, A. (Eds.). Mejora de la calidad Educativa en México: Posiciones y propuestas. Consejo Mexicano de Investigación Educativa. https://bit.ly/3a7hAvi

Prats, E. (2016). La formación inicial docente entre profesionalismo y vías alternativas: Mirada internacional. Bordón. Revista de Pedagogía, 68(2), 19-33. Doi: https://doi.org/10.13042/ bordon.2016.68202

Reynolds, D., Sammons, P., De Fraine, B., Townsend, T. \& Van Damme, J. (2011). Educational Effectiveness Research: A state of the art review. Paper presented to the annual meeting of the International Congress for School Effectiveness and Improvement. Cyprus. Doi: https://doi.org/ 10.1080/09243453.2014.885450

Said-Hung, E., Gratacós, G. \& Valencia, J. (2017). Factores que influyen en la elección de las carreras de pedagogía en Colombia. Educação e Pesquisa, São Paulo, 43(1), 31-48. Doi: http://dx.doi. org/10.1590/S1517-9702201701160978.

Tomás, J. (2014). Estudio de percepción, expectativas, aspiraciones y actitud de compromiso respecto a la carrera docente. Santo Domingo: MINERD. https://bit.ly/2QTmpAV
Topkaya, E. \& Uztosun, M. (2012). Choosing teaching as a career: Motivations of Pre-service English Teachers in Turkey. Journal of Language Teaching and Research, 3(1), 126-134. Doi: https:// doi.org/10.4304/jltr.3.1.126-134

Vaillant, D. \& Marcelo, C. (2012). Ensinando a ensinar: as quatro etapas de uma aprendizagem. Curitiba: Universidade Tecnologica Federal do Paraná. https://bit.ly/30gHAzT

Watt, H. \& Richardson, P. (2007). Motivational factors influencing teaching as a career choice: development and validation of the FIT-Choice scale. The Journal of Experimental Education, 75(3), 167-202. Doi: https://doi.org/10.3200/jexe.75. 3.167-202

Watt, H., Richardson, P., Klusmann, U., Kunter, M., Beyer, B., Trautwein, U. \& Baumert, J. (2012). Motivations for choosing teaching as a career: An international comparison using the FIT-Choice scale. Teaching and Teacher Education, 28(6), 791-805. Doi: https://doi.org/10.1016/j.tate. 2012.03.003

Wigfield, A. \& Eccles, J. (2000). Expectancy-Value Theory of Achievement Motivation. Contemporary Educational Psychology, 25(1), 68-81. Doi: https://doi.org/10.1006/ceps.1999.1015 